\title{
Avanços da maricultura na primeira década do século XXI: piscicultura e carcinocultura marinha
}

\author{
Luís André Sampaio ${ }^{1}$, Marcelo Borges Tesser², Wilson Wasielesky Júnior ${ }^{3}$ \\ ${ }^{1}$ Laboratório de Piscicultura Estuarina e Marinha - Universidade Federal do Rio Grande Instituto de Oceanografia. \\ ${ }^{2}$ Laboratório de Nutrição de Organismos Aquáticos - Universidade Federal do Rio Grande Instituto de Oceanografia. \\ ${ }^{3}$ Laboratório de Carcinocultura - Universidade Federal do Rio Grande Instituto de Oceanografia.
}

RESUMO - A piscicultura marinha é um setor pouco desenvolvido na maricultura brasileira. Por vários anos foi avaliado o potencial de cultivo de algumas espécies como o robalo-peva Centropomus parallelus e o linguado Paralichtys orbignyanus. Entretanto, somente a partir do investimento sobre o bijupirá Rachycentron canadum é que empresas privadas passaram a demonstrar maior interesse na atividade. Além dos sistemas tradicionais de piscicultura, o bijupirá pode ser criado em tanquesrede oceânicos. Esta espécie apresenta crescimento rápido, atingindo entre 4 e $8 \mathrm{~kg}$ em um ano de vida, e carne de excelente qualidade. A carcinocultura tem sido questionada por questões ambientais, uso de insumos como farinha e óleo de peixe e disseminação de doenças. A criação de camarões em sistemas sem renovação de água "ZEAH” (Zero Exchange, Aerobic, Heterotrophic Culture Systems) ou cultivo em meio aos Bioflocos (BFT) aplica métodos que minimizam estes problemas, contribuindo para uma maricultura mais saudável.

Palavras-chave: piscicultura, bijupirá, camarão

\section{Advances in mariculture on the first decade of the XXI century: marine fish and shrimp culture}

\begin{abstract}
Marine fish culture is still in its infancy in Brazil. For several years the snook Centropomus parallelus and the flounder Paralicithys orbignyanus were considered for aquaculture, but their commercial application has not yet been achieved. However, once technology for culture of cobia Rachycentron canadum became available, several private companies showed interest for marine fish culture. Besides traditional rearing technologes, cobia is suitable for open ocean culture in cages. This species shows fast growth rates, fish can achieve 4 or $8 \mathrm{~kg}$ within one year of age and its flesh is highly appreciated. Shrimp farming has been questioned for environmental issues, use of fish oil and fish meal, and spreading diseases. Rearing shrimp in systems without water exchange, know as ZEAH (Zero Exchange Aerobic Heterotrophic Culture systems) or bioflocs applies methods that minimize these problems, contributing for the development of sustainable shrimp farming.
\end{abstract}

Key Words: cobia, fish culture, shrimp

\section{Introdução}

O bijupirá Rachycentron canadum é a única espécie integrante da família Rachycentridae. São peixes pelágicos, migratórios, com ampla distribuição em águas tropicais e subtropicais. Estão presentes nos oceanos Atlântico, Índico e Pacífico, com exceção de toda costa leste do Oceano Pacífico e da costa européia (Shaffer \& Nakamura 1989). No Brasil, o bijupirá está presente em todo o litoral, mas é mais abundante em águas tropicais (Figueiredo \& Menezes, 1980).

O bijupirá atinge $68 \mathrm{~kg}$ até $2 \mathrm{~m}$ de comprimento, sua expectativa de vida é de 15 anos (Shaffer \&Nakamura, 1989). Esta espécie apresenta hábito carnívoro, é bastante apreciada na pesca esportiva (Kaiser \& Holt 2005) e tem

\footnotetext{
Corresponding author: sampaio@mikrus.com.br
}

grande aceitação na culinária, especialmente na cozinha asiática (Liao et al., 2004).

A produção comercial de bijupirá teve início partir dos anos 90 em Taiwan com o domínio da tecnologia para a produção de juvenis em larga escala (Liao et al., 2001). A sua elevada taxa de crescimento, ao redor de $6 \mathrm{~kg}$ ou mais em um ano (Chou et al., 2001; Liao et al., 2004; Weirich et al., 2004), despertou o interesse na sua produção em vários países como os Estados Unidos, México e Brasil (Schwarz et al., 2007). Entretanto, grande parte da produção ainda é concentrada na China e Taiwan, sendo a produção mundial de bijupirá igual a 23 mil toneladas.

Uma das grandes vantagens para a criação do bijupirá no Brasil é a existência de tecnologia desenvolvida para o seu cultivo no exterior. Isso permite eliminar várias etapas 
básicas de pesquisa. Entretanto, é importante adequar a tecnologia disponível para a realidade brasileira.

A introdução desta espécie na piscicultura marinha do Brasil é um marco para o desenvolvimento da atividade, pois além da pesquisa desenvolvida nos laboratórios brasileiros, houve uma adesão imediata da classe empresarial.

A carcinocultura mundial vem apresentando um crescimento expressivo nos últimos anos e com isso alguns problemas relacionados à poluição de águas através da emissão de efluentes sem tratamento, crescente demanda por farinha e óleo de peixe utilizados na formulação de rações e também a disseminação de doenças. Por outro lado os cultivos sem renovação de água "ZEAH” (Zero Exchange, Aerobic, Heterotrophic Culture Systems) ou cultivo em meio aos Bioflocos (BFT) vem de encontro com os novos conceitos de uma aquicultura responsável e ambientalmente correta, já que são realizados praticamente sem renovação de água e com aproveitamento dos microorganismos como alimento natural, o que reduz o uso de ração. O sistema BFT além de melhorar os índices de produtividade, se comparado com os sistemas tradicionais de cultivo, apresenta maior biossegurança, pois diminui trocas de água, e com isso evita doenças. É muito interessante perceber que este tipo de sistema de cultivo utiliza pouca água, o que representa uma diminuição na emissão de efluentes, podendo produzir $1 \mathrm{~kg}$ de camarão utilizando menos que 160 litros de água (Otoshi et al., 2006) enquanto nos sistemas convencionais é utilizado até 64.000 litros de água para produzir $1 \mathrm{~kg}$ de camarão (Hopkins et al., 1995). Um aspecto importante que deve ser considerado é que a formação dos agregados microbianos permite um melhor aproveitamento dos nutrientes originados da ração não consumida pelos camarões (Browdy et al., 2001; Burford et al., 2003), possibilitando a utilização de rações com menores teores de proteína bruta (Moss et al., 2001; Samocha et al., 2004).

\section{Piscicultura marinha: criação de bijupirá}

\section{Reprodução}

Em regiões subtropicais a reprodução ocorre nos meses de verão, enquanto em latitudes mais baixas o período de desova pode ser mais estendido (Shaffer \& Nakamura 1989). O bijupirá apresenta desova parcelada (Brown-Peterson et al., 2001) e de modo geral os machos atingem a maturidade com dois anos de vida $(65 \mathrm{~cm})$ e as fêmeas com três anos de vida $(80 \mathrm{~cm})$ na natureza (Franks \& Brown-Peterson, 2002). Entretanto, em cativeiro o bijupirá é mais precoce, sendo possível obter desovas de peixes com menos de dois anos de vida (Holt et al., 2007). A fecundidade relativa para cada desova é aproximadamente igual a $50 \mathrm{mil} \mathrm{ovos} / \mathrm{kg}$.
Combinando a fecundidade relativa com a duração do período reprodutivo e com a frequência de desovas (o intervalo de desovas varia de 5 a 12 dias), a fecundidade anual de uma fêmea com $20 \mathrm{~kg}$ foi estimada entre 8 e 40 milhões de ovos (Brown-Peterson et al., 2001).

A produção comercial de juvenis de bijupirá requer o domínio das técnicas de reprodução, incubação e larvicultura, de modo que a meta de produção de juvenis seja atingida sem oscilações. Além das desovas normalmente realizadas durante o período natural de reprodução é importante estabelecer um manejo adequado dos reprodutores para obter peixes sexualmente maturos ao longo de todo o ano.

A reprodução de bijupirá em cativeiro pode ser realizada por meio de indução hormonal (Caylor et al., 1994), mas a possibilidade de coletar ovos após desovas naturais de reprodutores mantidos em laboratório facilita a obtenção de ovos (Arnold et al., 2002; Benetti et al., 2008b).

Reprodutores de bijupirá podem ser coletados com linha e anzol. Não é possível determinar o sexo do bijupirá através de exame externo, por isso, logo após a coleta, os peixes devem ser mantidos sobre uma toalha úmida para que os gametas sejam coletados através de canulação (cânula com $3 \mathrm{~mm}$ de diâmetro externo e 1,5 mm de diâmetro interno). A coleta dos gametas permite a determinação do sexo e do estágio de maturação gonadal. Os machos são identificados com mais facilidade, pois normalmente liberam sêmen após uma leve pressão na região abdominal (Caylor et al., 1994). O transporte dos reprodutores pode ser feito em tanques com capacidade de 500 a $1.200 \mathrm{~L}$, durante o transporte a densidade de estocagem não deve exceder $50 \mathrm{~kg} / \mathrm{m}^{3}$. A concentração de oxigênio dissolvido não deve ser inferior a $7 \mathrm{mg} / \mathrm{L}$ e a temperatura deve ser mantida entre 22 e $24^{\circ} \mathrm{C}$.

Caylor et al. (1994) induziram a desova em fêmeas (peso entre 8 e $13 \mathrm{~kg}$ ) com ovócitos de diâmetro igual ou superior a $700 \mu \mathrm{m}$. A indução foi feita com injeção de HCG (gonadotropina coriônica humana, $275 \mathrm{UI} / \mathrm{kg}$ ). As fêmeas estavam aptas para extrusão manual dos ovócitos entre 76 e 90 h depois da indução. É interessante ressaltar que a nadadeira anal das fêmeas apresentava coloração avermelhada quando houve êxito na extrusão manual dos ovócitos e quando houve desova natural no tanque, sugerindo que esse seja um indicador do momento da ovulação.

A fertilização artificial não é o método mais popular para a produção de ovos de bijupirá. Entretanto, a indução hormonal da ovulação também tem sido utilizada para obtenção de desovas naturais em tanques e viveiros. Franks et al. (2001) obtiveram desova natural em tanques após induzir duas fêmeas com uma dose de HCG (275 UI/kg), 
quando o diâmetro médio dos maiores ovócitos era igual a $625 \mu \mathrm{m}$. Weirich et al. (2006) utilizaram implante intramuscular de GnRHa (hormônio análogo ao hormônio liberador de gonadotropina, 14,6 $\mu \mathrm{g} / \mathrm{kg}$ ) em reprodutores que posteriormente desovaram naturalmente em viveiros.

Reprodutores capturados na natureza podem ser aclimatados ao cativeiro e produzir ovos fertilizados naturalmente (Arnold et al., 2002). Entretanto, deve ser buscado um programa de domesticação da espécie, com o uso de reprodutores produzidos em cativeiro, de modo que o melhoramento genético possa ser realizado. Holt et al. (2007) e Benetti et al. (2008b) obtiveram sucesso na obtenção de ovos a partir de reprodutores nascidos em cativeiro (F1).

Ao trabalhar com reprodutores selvagens é importante adotar medidas profiláticas. Logo após chegar ao laboratório os reprodutores devem receber um banho de água doce por 2 min e em seguida um banho de formalina (100 ppm) com duração de $3 \mathrm{~min}$, com o objetivo de eliminar parasitas externos. Algumas lesões ocorridas durante a captura ou o transporte dos reprodutores podem proporcionar o desenvolvimento de infecções. Nesse caso é recomendado o tratamento destes peixes com banhos diários de oxitetraciclina ( 50 ppm) com duração de 3 h durante 5 dias. Um período de quarentena de até duas semanas é recomendado antes que os novos reprodutores sejam estocados nos tanques de maturação (Benetti et al., 2008b).

A densidade de estocagem para os reprodutores de bijupirá deve ser de no máximo $2 \mathrm{~kg} / \mathrm{m}^{3}$, com peixes pesando entre 3 e 20 kg (Arnold et al., 2002; Benetti et al., 2008b). O número de machos deve ser preferencialmente maior do que o de fêmeas, atingindo uma proporção de dois machos para cada fêmea (Benetti et al., 2008b).

Os reprodutores de bijupirá são alimentados basicamente com alimento fresco, mas eventualmente dietas artificiais também são oferecidas em complementação. Arnold et al (2002) utilizaram pescado descartado da pesca de camarão, principalmente os peixes: Leiostomus xanthurus, Lagodon rhomboid e Micropogonias undulatus. Duas vezes por semana esta dieta era suplementada em $20 \%$ com lula e camarão. A taxa de arraçoamento diário variou entre 2 e $3 \%$ do peso vivo por dia. Benetti et al (2008b) utilizaram alimento recém descongelado, principalmente peixes (Clupeidae e Carangidae) e lulas. O alimento era oferecido seis vezes por semana, sendo a taxa de arraçoamento de 2 a $4 \%$ do peso vivo por dia. As duas dietas propostas proporcionam taxas de fertilização semelhantes, podendo variar entre 40 e $95 \%$.

O tamanho dos tanques de maturação também tem influência sobre o sucesso da reprodução, uma vez que este pode interferir no comportamento de coorte no momento da desova. O formato, área e volume dos tanques para manutenção de reprodutores de bijupirá pode ser variável. Arnold et al. (2002) obtiveram ovos fertilizados em tanques semi-ovais com comprimento de 7,5 $\mathrm{m}$ largura de $4,5 \mathrm{~m} \mathrm{e}$ altura de 1,1 m com apenas $35 \mathrm{mil} \mathrm{L}$ de água em um sistema de recirculação de água. Por sua vez, Benetti et al. (2008a) utilizaram tanques circulares de 80 mil L, também com água recirculada. Já no Brasil, a equipe da AquaLider vem atingindo sucesso na produção de ovos fertilizados em tanques circulares de $7 \mathrm{~m}$ de diâmetro e $2 \mathrm{~m}$ de altura com fluxo contínuo de renovação de água (Manoel Tavares, com. pes.).

O sistema de recirculação de água utilizado para a manutenção dos reprodutores de bijupirá deve ser o mais completo possível. A água que saí do tanque pode ser direcionada para um tanque de sedimentação e dali imediatamente para um filtro mecânico onde o material particulado é removido. Em seguida, a água pode passar por um filtro tipo "skimmer" para remoção de matéria orgânica dissolvida, filtro biológico para remoção de compostos nitrogenados tóxicos e lâmpada ultra-violeta para esterilização. O desenho e o dimensionamento de cada sistema devem ser projetados para cada laboratório, em função das particularidades de cada região e do recurso disponível.

A desova natural do bijupirá é controlada pelo fotoperíodo e pela temperatura da água. Arnold et al. (2002) obtiveram as primeiras desovas quando a temperatura ultrapassou os $24^{\circ} \mathrm{C}$ e o fotoperíodo atingiu 13 horas de luz por dia. Temperaturas acima de $28^{\circ} \mathrm{C}$ parecem inibir a desova de bijupirá. A mesma combinação de temperatura e fotoperíodo foi utilizada com sucesso por Benetti et al (2008b) para a obtenção de ovos fertilizados de bijupirá. A temperatura não deve ser inferior a $19^{\circ} \mathrm{C}$ no inverno e deve haver uma elevação de aproximadamente $1^{\circ} \mathrm{C}$ por mês, até que no verão a temperatura esteja entre 26 e $28^{\circ} \mathrm{C}$ (Kaiser \& Holt, 2005).

Uma descrição interessante do comportamento de desova do bijupirá foi feito por Arnold et al. (2002). Normalmente os reprodutores nadam vagarosamente contra a corrente junto à parede do tanque. Entretanto, no dia em que ocorrem as desovas é observada uma maior atividade dos peixes no tanque de maturação. Aparentemente um casal é formado e estes dois peixes passam a nadar juntos, afastando os demais peixes presentes no tanque. Apesar de aparentemente apenas um casal participar da desova, os peixes comem com menos voracidade no dia da desova. Esta mudança de comportamento pode permitir prever a ocorrência de desova, que normalmente ocorre no início da noite. 
Os ovos fertilizados de bijupirá possuem flutuabilidade positiva em água salgada. Essa característica facilita o manejo da desova, pois os ovos fertilizados podem ser facilmente retirados do tanque de maturação através de um sistema de coleta de ovos. Este sistema é composto por um filtro tipo "skimmer" de superfície que leva os ovos por gravidade até um tanque externo onde os ovos são retidos em uma tela de $800 \mu \mathrm{m}$ (Kaiser \& Holt, 2005).

Além do sucesso na produção de ovos fertilizados em tanques, também foi observado desova de bijupirá em viveiros externos, tanto em viveiros de terra (400-600 $\mathrm{m}^{2} \mathrm{e}$ $1,5 \mathrm{~m}$ de profundidade) (Liao et al., 2004), como em viveiros circulares forrados com geomembrana $\left(2.500 \mathrm{~m}^{2}\right)$ (Weirich et al., 2006). Nestes casos os ovos podem ser coletados na superfície da água e levados para incubadoras ou podem ser deixados para eclodir no viveiro. O sucesso na criação das larvas no viveiro depende da disponibilidade de alimento vivo, sendo possível produzir larvas no viveiro e uma vez que o processo de primeira alimentação esteja concluído elas podem ser transferidas para sistemas intensivos de larvicultura (Weirich et al., 2006).

\section{Incubação e larvicultura}

Os ovos podem ser transferidos do coletor para incubadoras ou diretamente para os tanques de larvicultura. Larvas recém eclodidas são frágeis e para evitar a sua manipulação seria interessante estocar os ovos diretamente no tanque de larvicultura. Entretanto, a desvantagem deste método é a presença de ovos não eclodidos e do córion nos tanques de larvicultura, que podem ser substrato para o desenvolvimento de microorganismos indesejáveis.

Independente do sistema de incubação adotado é importante tratar os ovos para combater fungos e bactérias presentes na sua superfície. Hitzfelder et al. (2006) trataram os ovos de bijupirá com $1 \mathrm{ppm}$ formalina durante $10 \mathrm{~min}$, enquanto Benetti et al (2008b) recomendam um tratamento mais forte, com 100 ppm de formalina durante 30 minutos antes da transferência dos ovos.

A incubadora para ovos de bijupirá pode ter formato cilindro-cônico, com forte aeração proveniente de um anel de aeração colocado no fundo da incubadora. Um sistema de fluxo contínuo de renovação de água é utilizado, com troca diária de $1000 \%$ do volume em uma incubadora de 1.000 L. A transferência dos ovos do coletor para a incubadora pode ser feita durante a noite, logo após a desova, quando os ovos estão entre o estágio de 8 e 128 células (2-3 h após a fertilização). Alternativamente, os ovos podem ser transferidos pela manhã, já em um estágio mais avançado do desenvolvimento embrionário (12 h após a fertilização). A densidade de estocagem pode ser mantida entre 100 e 500 ovos/L (Benetti et al., 2008b).

Ovos de bijupirá possuem diâmetro de 1,35-1,40 mm (Liao et al., 2001). Holt et al. (2007) constataram aumento no diâmetro do ovo ao reduzir a salinidade da desova de 34 para 28\%o. Entretanto, isso não se refletiu em um aumento no comprimento das larvas ao eclodir. O tempo para eclosão é de aproximadamente $24 \mathrm{~h}$ a $28^{\circ} \mathrm{C}$ (Benetti et al. 2007) e o comprimento total das larvas ao eclodir é de 3,5 mm (Liao et al., 2001).

A larvicultura de bijupirá é realizada com temperatura entre 28 e $32^{\circ} \mathrm{C}$ em água salgada, mantendo a concentração de amônia total abaixo de $0,1 \mathrm{mg} / \mathrm{L}$ e o pH ao redor de 8 , com auxílio de renovação de água entre 100 e $600 \%$ por dia ao longo dos primeiros 30 dias de vida. O fotoperíodo é o natural do verão, ao redor de 13 a 14 h de luz por dia (Benetti et al., 2008a).

Vários protocolos alimentares foram sugeridos para a larvicultura de bijupirá. O protocolo descrito a seguir permite a produção de juvenis de bijupirá com sobrevivência de $25 \%$ e peso de $1,8 \mathrm{~g}$ aos 35 dias de vida. É necessário estimar a densidade de presas a cada $6 \mathrm{~h}$ e repor a quantidade necessária para manter a densidade desejada. A primeira alimentação é oferecida dois dias após a eclosão (DAE) e a presa oferecida como primeiro alimento é o rotífero Brachionus plicatilis na densidade de 2 indivíduos $/ \mathrm{mL}$. Rotíferos são oferecidos em conjunto com a microalga Nannochloropsis (densidade de 120 mil células/mL) até o oitavo DAE, quando então a oferta dos dois é suspensa. Durante três dias, a partir do sexto DAH são oferecidos náuplios de Artemia AF (tipo pequeno) na densidade de 1 indivíduo/mL. A partir do oitavo DAE passa a ser oferecido náuplio de Artemia EG (tipo grande) sem enriquecimento, a densidade deve aumentar ao longo destes cinco dias de 1,5 para 3,5 individuos/mL. Artemia enriquecida passa a ser oferecida a partir do $12^{\circ} \mathrm{DAE}$ e continua sendo oferecida até o $25^{\circ}$ DAE, a densidade de presas aumenta de 1,5 para $3,5 / \mathrm{mL}$ durante este período. A co-alimentação de Artemia com dietas inertes tem início no $18^{\circ}$ DAE. A partir do $25^{\circ} \mathrm{DAE}$ a oferta de alimento vivo deve ser suspensa e o desmame completado. A partir deste momento apenas dietas inertes são oferecidas para os juvenis de bijupirá.

Durante a alimentação com alimento vivo, especialmente enquanto rotíferos são utilizados, é importante adicionar microalgas no tanque de larvicultura, uma técnica conhecida por “água verde”. Diversas espécies de microalgas podem ser utilizadas, mas Nannochloropsis vem sendo utilizada com sucesso na larvicultura de bijupirá. A produção de microalgas exige estrutura e pessoal e a possibilidade de 
utilizar microalgas congeladas, disponíveis no mercado, pode reduzir a atividade no laboratório. A comparação da performance dos juvenis produzidos com microalgas vivas ou congeladas (densidade de 120 mil células $/ \mathrm{mL}$ ) mostrou que não há qualquer comprometimento pelo uso das microalgas congeladas em termos de sobrevivência e crescimento do bijupirá (Schwarz et al., 2008).

Várias densidades de estocagem foram avaliadas para a larvicultura de bijupirá com o objetivo de otimizar crescimento e número de juvenis produzidos. Hitzfelder et al. (2006) recomendam a densidade inicial de 5 a 10 larvas/L para atingir a densidade de estocagem final ao redor de 1 juvenil/L após 21 dias de cultivo.

No sistema de produção de Taiwan, é registrada uma grande variação no tamanho do bijupirá após 18-25 dias de vida e pode haver incidência de canibalismo. Para reduzir o canibalismo os peixes são classificados por tamanho a cada 3-4 dias, até completarem 35-45 dias de vida (Liao et al., 2001). A ocorrência de canibalismo em juvenis de bijupirá é sustentada pela observação de Faulk et al. (2007), a sobrevivência de peixes que não foram gradeados foi menor do que a sobrevivência daqueles criados em tanques onde foi feito o gradeamento, mas nenhum indivíduo morto foi encontrado nos tanques não gradeados.

A larvicutura de bijupirá tem sido realizada também no sistema semi-intensivo. Esse sistema caracteriza-se pela fertilização prévia dos viveiros e a estocagem de larvas quando as mesmas abrem a boca (48-72 h após a eclosão). Weirich et al. (2004) relatam a larvicultura nesse sistema utilizando tanques recobertos com geomembrana e área de $2.500 \mathrm{~m}^{2}$, onde as larvas são alimentadas com ração suplementar com quantidades e granulometrias crescentes. Resultados variados foram obtidos, o que pode ser explicado pela falta de controle sobre a população planctônica produzida, presença de predadores e sobre a qualidade de água. Assim no ano de 2001 foram estocadas 700.000 larvas/ha e após cinco semanas a sobrevivência foi em torno de $5-8,5 \%$ e juvenis atingiram aproximadamente $9 \mathrm{~g}$ de peso.

\section{Engorda}

Os juvenis para a fase de engorda do bijupirá em Taiwan são obtidos por um sistema dividido em três fases. Esta etapa é realizada em viveiros, pois apesar da preferência de bijupirá por água limpa, eles toleram bem água verde especialmente durante o primeiro ano de vida (Chang et al., 2007).

A primeira fase tem início com animais de $0,2 \mathrm{~g}$ e termina com peixes com $5 \mathrm{~g}(8-10 \mathrm{~cm})$ e tem duração de aproximadamente 25 dias. Durante essa fase os peixes são condicionados a comerem ração. Ao contrário do sistema intensivo americano em que o desmame (substituição do alimento vivo por alimento inerte) é completado ao redor de 25 dias de vida, no Taiwan o desmame é mais tardio. Na segunda fase os peixes são criados de 2 a $30 \mathrm{~g}$ em 30 dias. O alimento é fornecido seis vezes ao dia na taxa de arraçoamento de $5 \%$ da biomassa. A terceira etapa (de 30 até $600-1000 \mathrm{~g}$ ) tem duração de 75 dias e é feita em viveiros ou em tanques rede junto à costa. A criação de juvenis de bijupirá menores de $30 \mathrm{~g}$ em tanques em mar aberto não é recomendado devido a baixa resistência desses peixes à fortes correntezas (Liao et al., 2004).

Juvenis de bijupirá foram criados em sistema de recirculação de água para posteriormente serem transferidos para tanques-rede em mar aberto. Peixes com peso inicial de $7 \mathrm{~g}$ foram estocados na densidade de $2,7 \mathrm{~g} / \mathrm{L}$ e atingiram em torno de $90 \mathrm{~g}$ após quatro semanas (Liao et al., 2004). Entretanto, Webb et al. (2007) sugerem que melhor crescimento pode ser obtido ao utilizar uma densidade de estocagem menor $(0,44 \mathrm{~g} / \mathrm{L})$ para peixes deste mesmo tamanho.

A engorda de bijupirá é feita na maioria dos casos em sistemas intensificados como tanques-rede e sistemas de recirculação. Em Taiwan dois modelos são utilizados quanto à produção em tanques-rede, o familiar e o industrial (Liao et al., 2004). No sistema familiar os peixes passam por quatro tanques diferentes. Juvenis com $10 \mathrm{~g}$ são estocados em tanques-rede retangulares de $27 \mathrm{~m}^{3}$ na densidade de $7,4 \mathrm{~kg} / \mathrm{m}^{3}$ por 2 ou 3 meses. Após esse período os peixes agora com $100 \mathrm{~g}$ em média são estocados em tanques maiores $\left(64 \mathrm{~m}^{3}\right)$ na densidade de $11 \mathrm{~kg} / \mathrm{m}^{3}$ e após 3-4 meses atingem 500 g. Os peixes são então transferidos para tanques circulares de $350 \mathrm{~m}^{3}$ na densidade de $14 \mathrm{~kg} / \mathrm{m}^{3} \mathrm{e}$ permanecem nesse tanque por 2-3 meses atingindo $2 \mathrm{~kg}$. O período final de engorda se dá em tanques de $1.000 \mathrm{~m}^{3}$, densidade de $14 \mathrm{~kg} / \mathrm{m}^{3}$ até atingirem o peso comercial de $6 \mathrm{~kg}$ por mais 4-5 meses. Por outro lado, o sistema industrial é baseado no uso de duas etapas e o peso inicial dos peixes é maior do que no sistema familiar. Peixes com peso médio de $30 \mathrm{~g}$ são estocados em tanques-rede circulares de $190 \mathrm{~m}^{3}$ na densidade de $8,4 \mathrm{~kg} / \mathrm{m}^{3}$. Quando os peixes atingem cerca de $800 \mathrm{~g}$ eles são transferidos para tanquesrede maiores $\left(1.800 \mathrm{~m}^{3}\right)$ na densidade de $14 \mathrm{~kg} / \mathrm{m}^{3}$ até atingirem o peso comercial entre 6 e 8 meses.

O uso de tanques-rede submersíveis de $25 \mathrm{~m}$ de diâmetro e $15 \mathrm{~m}$ de profundidade $\left(2.700 \mathrm{~m}^{3}\right)$ é eficiente para a produção de bijupirá. Um ciclo de criação iniciado com juvenis de $1,5 \mathrm{~g}$ resultou em peixes com $6 \mathrm{~kg}$ após 12 meses, com conversão alimentar 2 e sobrevivência acima de $75 \%$. O 
crescimento final dos bijupirás foi dependente da densidade, pois o aumento da densidade de estocagem de 5 para $15 \mathrm{~kg} / \mathrm{m}^{3}$ resulta em menor crescimento com consequente piora no valor de conversão alimentar (Benetti et al., 2007).

Miao et al. (2009) estudaram por meio de modelos econômicos e matemáticos a produção de bijupirás em Taiwan. Esses autores concluíram que para maximizar os lucros as fazendas devem ser grandes. Os autores discutem ainda sobre uma possível influência das velocidades de correntes dos diferentes ambientes sobre o lucro obtido, pois há uma relação inversa entre a velocidade da corrente e o lucro, desde que a velocidade seja suficiente para manter a qualidade da água.

A experiência de engorda do bijupirá no Brasil ainda é muito limitada. Avaliações preliminares vêm sendo feitas no litoral das regiões sudeste e nordeste e a principal constatação feita até o momento remete para a necessidade de produção de dietas de melhor qualidade. Rombenso et al. (2009) compararam o crescimento de juvenis de bijupirá alimentados com uma dieta comercial ou com sardinha congelada e observaram que os peixes alimentados com sardinha atingiram $200 \mathrm{~g}$, enquanto aqueles alimentados com uma dieta comercial atingiram apenas $90 \mathrm{~g}$ após 2 meses de crescimento em tanque-rede. Juvenis de bijupirá com peso inicial de 1,5 $\mathrm{g}$ foram criados durante um ano em tanque-rede no litoral de Angra dos Reis e atingiram o peso médio de 4,2 kg, mas foram observados indivíduos com até $6 \mathrm{~kg}$ (Sampaio et al., 2009).

\section{Qualidade da água}

O controle da qualidade da água, através da manutenção de condições adequadas de temperatura, salinidade, $\mathrm{pH}$, oxigênio e compostos nitrogenados é importante para manter os peixes saudáveis.

Temperaturas entre 27 e $29^{\circ} \mathrm{C}$ são as mais adequadas para otimizar o crescimento e a eficiência alimentar de juvenis de bijupirá (Sun et al., 2006). OS peixes podem ser mantidos em temperaturas mais baixas (até $18^{\circ} \mathrm{C}$ ) para retardar propositadamente o crescimento, ao elevar a temperatura para condições de crescimento acelerado os juvenis de bijupirá apresentam crescimento compensatório (Schwarz et al., 2007). Carvalho et al. (2006) estudaram a temperatura letal superior para juvenis de bijupirá. Foi observado que os peixes cessam sua alimentação entre 36,5 e $37,5^{\circ} \mathrm{C}$, perdem o equilíbrio entre 36 e $37^{\circ} \mathrm{C}$ e atingem a temperatura letal superior para $50 \%$ da população em $36,5^{\circ} \mathrm{C}$.

Os juvenis de bijupirá mostram tolerância a salinidades baixas, Atwood et al. (2004) observaram mortalidade total de juvenis em salinidade abaixo de 2. Denson et al. (2003) observaram maior crescimento de juvenis criados em salinidade 30 do que nas salinidades 5 e 15 . Entretanto, Resley et al. (2006) não encontraram diferenças no crescimento de juvenis de bijupirá criados entre as salinidades 5 e 30 .

A amônia e o nitrito são compostos reconhecidamente tóxicos para peixes, mesmo em baixas concentrações. Juvenis de bijupirá cessam a alimentação quando a concentração de amônia atinge $0,62 \mathrm{mg} \mathrm{N}-\mathrm{NH}_{3} / \mathrm{L}$ e a mortalidade para $50 \%$ da população é observada $1,13 \mathrm{mg}$ $\mathrm{N}-\mathrm{NH}_{3} / \mathrm{L}$. Estas concentrações de amônia podem ser facilmente encontradas em sistemas de cultivo intensivo e especial cuida deve ser tomado quando o $\mathrm{pH}$ for elevado, situação em que ocorre uma maior proporção de amônia gasosa do que iônica, portanto o pH deve ser mantido abaixo de 8 . Por outro lado, os juvenis de bijupirá são bastante resistentes ao nitrito e apenas 30\% de mortalidade é observada quando a concentração atinge $210 \mathrm{mg} \mathrm{N}-\mathrm{NO}_{2} / \mathrm{L}$ e a alimentação é interrompida em $76 \mathrm{mg} \mathrm{N}-\mathrm{NO}_{2} / \mathrm{L}$ (Rodrigues et al., 2007).

A performance de bijupirá criado em $\mathrm{pH} 8$ ou 7 é superior a dos peixes criados em $\mathrm{pH}$ mais reduzido. O crescimento e a eficiência de conversão alimentar são comprometidos quando os juvenis de bijupirá são criados em sistemas de recirculação de água com pH 6 ou 5 (Sampaio et al., 2008). Cultivo de camarões marinhos em sistema de bioflocos

\section{Histórico da produção em sistema de bioflocos}

O cultivo de camarões marinhos nas Américas pode ser dividido em três períodos, se levarmos em consideração as estratégias de cultivo utilizadas. O primeiro deles (anos 80/90) foi marcado pelas grandes áreas de cultivo em viveiros utilizando baixas densidades de estocagem. Neste período o país com maior produção foi o Equador, com produtividades médias em torno de $300 \mathrm{~kg} / \mathrm{ha} /$ ciclo. O segundo período, nos anos noventa, caracterizou-se também pelos cultivos em viveiros utilizando, porém, mais tecnologia, como é o caso do Brasil, com produtividades acima de $2.000 \mathrm{~kg} / \mathrm{ha} /$ ano. Enquanto isso, nos Estados Unidos da América, Hopkins et al. (1995) iniciaram pesquisas para o desenvolvimento de tecnologias ambientalmente amigáveis com objetivos de diminuir a emissão de efluentes com produtividades também acima de $5.000 \mathrm{~kg} / \mathrm{ha} /$ ciclo. Apesar dos esforços, o surgimento de doenças como a Síndrome de Taura, Mancha Branca, entre outras, prejudicou a atividade em diferentes países americanos (Wasielesky et al., 2006). Em decorrência, nos últimos anos, surgiu o terceiro momento dos cultivos de camarões, com o uso de novas tecnologias desenvolvidas, principalmente, nos Estados Unidos (Browdy et al., 2001) e em Belize (Burford et al., 2003), com objetivo de 
produzir camarões em sistemas fechados, ou seja, cultivo de camarões em sistemas de bioflocos.

McAbee et al. (2003) no Centro de Maricultura de Waddell (EUA) utilizando densidades de estocagem de 300 camarões $/ \mathrm{m}^{2}$ obtiveram biomassa final de $3 \mathrm{~kg} / \mathrm{m}^{2} / \mathrm{safra}$ e sobrevivência de $70 \%$ na fase de engorda. Neste mesmo centro de pesquisa, no ano 2004 foram atingidas produtividades acima de $5,0 \mathrm{~kg} / \mathrm{m}^{2} / \mathrm{safra}$ em raceways com densidades iniciais acima de $400 / \mathrm{m}^{2}$, o que equivaleria a 50 ton/ha/ciclo, ou seja, de 10 a 20 vezes o que se produz em sistemas convencionais.

\section{A formação dos bioflocos}

A formação dos bioflocos ocorre a partir da mudança da razão entre carbono e nitrogênio $(\mathrm{C}: \mathrm{N})$ dos cultivos. Esta deve manter-se entre 15 e 20:1, para que ocorra o surgimento de bactérias heterotróficas, e com isso inicie toda uma sucessão microbiana até a formação dos bioflocos. Para isto se faz aplicações de melaço de canade-açúcar e/ou dextrose como fonte de carbono. A partir da mudança desta relação $\mathrm{C}: \mathrm{N}$ e através de uma forte aeração, macro agregados ou "flocos" são formados durante o ciclo de produção. Sendo constituídas principalmente de bactérias, microalgas, fezes, exoesqueletos, restos de organismos mortos, protozoários, invertebrados, entre outros. Uma vez formados, eles servem de suplemento alimentar aos animais, bem como assimilarão compostos nitrogenados presentes na água de cultivo. Outro fator de suma importância associado à formação desses agregados e ao incremento na produtividade primária é o fato da utilização de menores teores de proteína bruta nas rações, sendo agora em parte suprida pela produção natural, acarretando em diminuição nos custos do produtor, além de ser ambientalmente correto (Wasielesky et al., 2006). Estudos realizados em Belize demonstraram que mais de $29 \%$ do alimento consumido por Litopenaeus vannamei podem ser provenientes dos flocos microbianos (bioflocos) presente na água do cultivo, demonstrando assim a viabilidade do sistema (Burford et al., 2003).

Vantagens do tipo de sistema proposto em relação ao sistema tradicional

Os sistemas tradicionais de cultivo de camarão se caracterizam por ocupar grandes áreas territoriais quase que exclusivamente em regiões costeiras, para manter a qualidade da água necessitam de altas taxas de renovação liberando seus efluentes carregados de nutrientes diretamente no ambiente. Além disso, a ração que não é consumida pelos camarões é eliminada durante esta renovação de água. Por outro lado, os cultivos em meio heterotrófico podem ser realizados em áreas relativamente pequenas, pois utilizam altas densidades de estocagem, e como este sistema não possui renovação de água não ocorre liberação de nutrientes no ambiente. Além disso, pode ser realizado em regiões distantes da costa. Neste sistema a ração não consumida é reciclada e entra novamente no sistema na forma de proteína microbiana sendo então consumida pelos camarões.

\section{Densidade de estocagem}

No Brasil, inicialmente realizaram-se testes em berçários intensivos com densidades entre 1500 e 6000 camarões/ $\mathrm{m}^{2}$. Os resultados obtidos são estimuladores, pois as sobrevivências foram acima de 90\% em diferentes densidades, sem renovação de água (Froes, 2007). Na fase de engorda, Krummenauer (2008) testou as densidades de 150,300 e 450 camarões $/ \mathrm{m}^{2}$ durante 90 dias (a partir de $1 \mathrm{~g}$ ). Os melhores resultados foram observados na densidade de $300 / \mathrm{m}^{2}$, com crescimento semanal de $0,82 \mathrm{~g}$, sobrevivência acima de $85 \%$ e taxa de conversão de 1,3 . A produtividade foi $3,9 \mathrm{~kg}$ de camarões por $\mathrm{m}^{2}$ (39 toneladas/ ha/ciclo). Silva (2009) realizando testes de engorda com camarões a partir de $12 \mathrm{~g}$ nas densidades de 150, 300, 450 e $600 / \mathrm{m}^{2}$ obteve resultados para biomassa final de $5,1 \mathrm{~kg} / \mathrm{m}^{2}$ (51 ton/ha/ciclo), mas com sobrevivência de apenas 75,1\%. Nas densidades de $300 / \mathrm{m}^{2}$ as sobrevivências têm sido acima de $85 \%$ em ciclos de 90 a 120 dias. Os resultados evidenciam que a densidades de estocagens recomendadas para o cultivo de L. vannamei no sistema proposto podem ser entre 300 e 350 camarões $/ \mathrm{m}^{2}$. Os valores obtidos são menores que aqueles registrados dos sistemas utilizados nos Estados Unidos, entretanto utilizando menores recursos tecnológicos do que as empregadas naquele país e com menores custos operacionais.

\section{Cultivo em raceways}

O cultivo de camarões em raceways cobertos (estufas) tem despertado interesse de pesquisadores e produtores em alguns países do mundo, oportunizando o cultivo de camarões peneídeos em regiões com clima sub-tropical e/ou temperado. Nos Estados Unidos da América (Carolina do Sul, Virgínia, Maryland, Texas, Havaí, entre outros, muitas pesquisas tem sido realizadas para a produção em estruturas fechadas (raceways em estufas). As principais vantagens estão associadas à proximidade do mercado, além daquelas citadas na Tabela 1 . Na Coréia do Sul, Indonésia, Bélgica e Holanda, sistemas BFT também já estão sendo utilizados para a engorda de camarões. utilizando água quente de indústria.

No ano de 2005, pesquisadores do Waddell Mariculture Center(Carolina do Sul, EUA)(Browdy, com.pess.) atingiram 
Tabela 1 - Síntese das vantagens e desvantagens dos cultivos de camarões em sistemas de bioflocos

\begin{tabular}{ll}
\hline \multicolumn{1}{c}{ Vantagens } & \multicolumn{1}{c}{ Desvantagens } \\
\hline Aumento da produtividade \\
Utilização de menores áreas de cultivo \\
$\begin{array}{l}\text { Aumento da biossegurança } \\
\text { Diminuição ou isenção da renovação de água } \\
\text { Maior estabilidade do sistema } \\
\text { Diminuição da quantidade de proteína nas rações } \\
\begin{array}{l}\text { Maior disponibilidade de alimento natural } \\
\text { Comunidade microbiana atuando com probiótico } \\
\text { Menores unidades de cultivo com maior controle } \\
\text { Menor impacto ambiental }\end{array}\end{array}$ & Maiores gastos de energia (aerão) \\
\end{tabular}

produtividades de $6,8 \mathrm{~kg} / \mathrm{m}^{2} /$ ciclo em raceways com densidades iniciais acima de $400 / \mathrm{m}^{2}$ ( 68 ton $/$ ha). No ano de 2008 no Oceanic Institute (Havaí, EUA), Otoshi et al. reportaram produção de $10,3 \mathrm{~kg} / \mathrm{m}^{2}$ (103 ton/ha) com densidade inicial de 828 camarões $/ \mathrm{m}^{2}$ e densidade final de 562 camarões $/ \mathrm{m}^{2}$. Recentemente, Correia (UFRPE-Brasil) e Samocha (Texas A\&M-EUA) (2010) reportaram a produção em raceways de $9,75 \mathrm{~kg} / \mathrm{m}^{3}$ em raceways em Corpus Christi (Texas, EUA) com densidade inicial de 530 camarões $/ \mathrm{m}^{3}$.

É importante ressaltar que os resultados acima obtidos foram realizados com uso de altos recursos tecnológicos como oxigênio injetável, filtros biológicos, filtros mecânicos, fracionadores, sedimentadores, sistemas automatizados, e em alguns casos, com monitoramento eletrônico de qualidade da água. Além disso, a utilização de rações específicas para camarões em sistema superintensivos (BFT) provavelmente contribuiu para estes resultados.

No Sul do Brasil (Rio Grande do Sul), estudos com cultivo de camarões no sistema BFT têm sido realizados com objetivo de desenvolver pacotes tecnológicos de cultivo em raceways (em estufas) para as condições brasileiras. Nos últimos seis anos foram realizados estudos sobre os cultivos em sistema BFT em estufas de engorda. Foram realizados testes para determinação densidades de estocagem ideais; povoamento direto e sistema utilizando berçário, com objetivo paralelo de detectar crescimento compensatório; uso de probióticos; fertilização orgânica; diferentes sistemas de aeração; entre outros trabalhos (Emerenciano et al. 2007; Ballester et al., 2010; Silva, 2009; Krummenauer, 2008; Froes, 2007).

Os experimentos com raceways têm apresentado resultados animadores, demonstrando que a técnica é uma realidade e está pronta a ser aplicada em cultivos comerciais no país. A síntese dos resultados zootécnicos obtidos em viveiros de cultivo utilizando o sistema de flocos microbianos (BFT) no Rio Grande do Sul é apresentada na Tabela 2 .
Tabela 2 - Desempenho médio do camarão-branco L. vannamei em raceways com sistema BFT, nas instalações da Estação Marinha de Aquacultura (EMA/IO/FURG)

\begin{tabular}{lc}
\hline Densidade inicial & $400\left(\mathrm{ind} / \mathrm{m}^{2}\right)$ \\
\hline Sobrevivência & $85,0(\%)$ \\
Ganho de peso/semana & $0,85(\mathrm{~g})$ \\
Peso médio inicial & $\mathrm{Juvenis} \mathrm{de} 1 \mathrm{~g}$ \\
Peso médio final & $15,57(\mathrm{~g})$ \\
Tempo médio de cultivo & $120(\mathrm{dia})$ \\
Biomassa final & $4632\left(\mathrm{~g} / \mathrm{m}^{2}\right)$ \\
Ração fornecida & $5512\left(\mathrm{~g} / \mathrm{m}^{2}\right)$ \\
Conversão alimentar & $1: 1,19$ \\
Produtividade & $46.321(\mathrm{~kg} / \mathrm{ha}) *$ \\
\hline
\end{tabular}

* Média dos resultados em 30 ciclos de cultivo em raceways de 50-100 $\mathrm{m}^{3}$ (100 hp/ha), revestidos com PEAD em estufas.

\section{Perspectivas Futuras}

Estima-se que os cultivos em raceways (com estufas, no sul do Brasil) no sistema BFT seja uma alternativa viável a ser aplicada em diferentes locais em função de ocupar áreas muito pequenas. Os resultados apresentados mostram que é possível se trabalhar com produtividades acima de 46 ton/ciclo ou acima de 130 t/ano.

Atualmente em nosso país contamos com uma série de elementos fundamentais para o tipo de sistema proposto. Entre estes podemos citar pós-larvas SPF (livre de patógenos), desenvolvimento genético, tecnologia de cultivo, geomembranas (PEAD), equipamentos, fontes de carbono de baixo custo, etc. Paralelamente a isto, existe também todo um "know-how" e uma cadeia produtiva da Carcinicultura bem definida. Sendo assim, estima-se que a aplicação desta nova tecnologia (cultivo em Bioflocos) seja relativamente de fácil implantação, através de alterações de sistemas já existentes. Consideramos que as maiores dificuldades para implementação desses sistemas no país serão principalmente o nível de conhecimento das pessoas, as quais devem se adaptar ao novo tipo manejo para cultivar camarões em sistema BFT. 


\section{Agradecimentos}

À FURG, MPA e CNPq (Processos 483433/2007-1; 559741/2009), pelo apoio financeiro. Ao CNPq, pela Bolsa de Produtividade em Pesquisa concedidas a Luís André Sampaio e Wilson Wasielesky Junior.

\section{Referências}

ARNOLD, C.R.; KAISER, J.B.; HOL, G.J. Spawning of cobia (Rachycentron canadum) in captivity. Journal of the World Aquaculture Society, v.33, p.205-208, 2002.

ATWOOD, H.L.; YOUNG, S.P.; TOMASSO, J.R. et al. Resistance of cobia, Rachycentron canadum, juveniles to low salinity, low temperature, and high environmental nitrite concentrations. Journal of Applied Aquaculture, v. 15, p.191-195, 2004.

BALlESTER, E.L.C.; ABREU, P.C.; CAVALLI, R. et al. Effect of practical diets with different protein levels on the performance of Farfantepenaeus paulensis juveniles nursed in a zero exchange suspended microbial flocs intensive system. Aquaculture Nutrition, v.16, p.163-172, 2010.

BENETTI D.D.; ORHUN M.R.; ZINK I.C. et al. Aquaculture of Cobia (Rachycentron canadum) in the Americas and the Caribbean. In: LIAO, I.C.; LEAÑO, E.M. (Eds.) Cobia aquaculture: research development and commercial production. Manila: Asian Fisheries Society, 2007. p.57-77.

BENETTI; D.D.; SARDENBERG, B.; WELCH, A. et al. Intensive larval husbandry and fingerling production of cobia Rachycentron canadum. Aquaculture, v.281, p.22-27, 2008a.

BENETTI; D.D.; REFIK ORHUN, M.; SARDENBERG, B. et al. Advances in hatchery and grow-out technology of cobia Rachycentron canadum (Linnaeus). Aquaculture Research, v.39, p.701-711, 2008b.

BROWDY, C.L.; BRATVOLD, D.; STOKES, A.D. et al. Perspectives on the application of closed shrimp culture systems. In: BROWDY, C.L.; JORY, D.E. (Eds.). The new wave. Baton Rouge: The World Aquaculture Society, 2001.

BROWN-PETERSON, N.J.; OVERSTREET, R.M.; LOTZ, J.M. et al. Reproductive biology of cobia, Rachycentron canadum, from coastal waters of the southern United States. Fisheries Bulletin, v.99, p.15-28, 2001.

BURFORD, M.A.; THOMPSON, P.J.; MCINTOSH, R.P. et al. Nutrient and microbial dynamics in high-intensity, zeroexchange shrimp ponds in Belize. Aquaculture, v.219, n.14, p.393-411, 2003.

CARVAlHo, C.V.A.; SILVA, T.J.; DElbos, B.C. et al. Temperatura letal superior para juvenis de bijupirá Rachycentron canadum. Anais... Bento Gonçalves: Aquaciência, 2006.

CAYLOR, R.E.; BIESIOT, P.M.; FRANKS, J.S. Culture of cobia Rachycentron canadum: cryopreservation of sperm and induced spawning. Aquaculture, v.125, p.81-92, 1994.

CHANG, S.L.; LIAO, I C., LEAÑO, E.M. Nursery rearing of Cobia in Taiwan: problems, recent developments and strategies. In: LIAO, I.C.; LEAÑO, E.M. (Eds.) Cobia aquaculture: research development and commercial production. Manila: Asian Fisheries Society, 2007. p.79-88.

COLBURN, H.R.; WALKER, A.B.; BERLINSKY, D.L. Factors effecting survival of cobia, Rachycentron canadum, during simulated transport. Journal of the World Aquaculture Society, v.39, p.678-683, 2008.

DENSON, M.R.; STUART, K.R.; SMITH, T.I.J. et al. Effects of salinity on growth, survival, and selected hematological parameters on juvenile cobia, Rachycentron canadum. Journal of the World Aquaculture Society, v.34, p.496$504,2003$.
EMERENCIANO, M.G.C.; WASIELESKY, W.; SOARES, R.B. et al. Crescimento e sobrevivência do camarão-rosa (Farfantepenaeus paulensis) na fase de berçário em meio heterotrófico. Acta Scientiarum Biological Sciences, v.29, n.1, p.1-7, 2007.

FAULK, C.K.; KAISER, J.B.; HOLT, J.G. Growth and survival of larval and juvenile cobia Rachycentron canadum in a recirculating raceway system. Aquaculture, v.270, p.149-157, 2007.

Figueiredo, J.L.; MeneZeS, N.A. Manual de peixes marinhos do sudeste do Brasil. III Teleostei (2). São Paulo: USP, 1980. 90p.

FRANKS, J.S.; BROWN-PETERSON, N.J. A review of age, growth, and reproduction of cobia, Rachycentron canadum, from U.S. waters of the Gulf of Mexico and Atlantic Ocean. Proceedings of the Gulf and Caribbean Fisheries Institute, v.53, p.552$569,2002$.

FRANKS, J.S.; OGLE, J.T.; LOTZ, J.M.et al. Spontaneous spawning of cobia Rachycentron canadum, induced by human chorionic gonadotropin (HCG), with comments on fertilization, hatching and larval development. Proceedings of the Gulf and Caribbean Fisheries Institute, v. 52, p.589-609, 2001.

FROES, C.N. Berçários intensivos em sistemas de bioflocos no sul do Brasil. 2007. Dissertação (Mestrado em Aquicultura) - Universidade Federal do Rio Grande, Rio Grande.

HITZFELDER, G.M.; HOLT, G.J.; FOX, J.M. et al. The effect of rearing density on growth and survival of cobia, Rachycentron canadum, larvae in a closed recirculating aquaculture system. Journal of the World Aquaculture Society, v.37, p.204209, 2006.

HOLT, G.H.; FAULK, C.K.; SCHWARZ, M.H. A review of the larviculture of cobia Rachycentron canadum, a warm water marine fish. Aquaculture, v.268, p.181-187, 2007.

HOPKINS, J.S.; SANDIFER, P.A.; BROWDY, C.L. A review of water management regimes which abate the environmental impact of shrimp farming. In: BROWDY, C.L.; HOPKINS, J.S. (Eds.) Swimming through troubled water. Baton Rouge: World Aquaculture Society, 1995.

KAISER, J.B.; HOLT, G.J. Species profile: Cobia. Southern Regional Aquaculture Center Publication, number 7202. (http:/ /www.ca.uky.edu/wkrec/Cobia.pdf), 2005.

KRUMMENAUER, D. Estratégias para o cultivo de Litopenaeus vannamei no extremo sul do Brasil. Dissertação (Mestrado em Aquicultura) - Universidade Federal do Rio Grande, Rio Grande, 2008.

LIAO, C.I.; HUNAG, T.S.; TSAI, W.S. et al. Cobia culture in Taiwan: current status and problems. Aquaculture, v.237, p.155-165, 2004.

LIAO, I.C.; SU, H.M.; CHANG, E.Y. Techniques in finfish larviculture in Taiwan. Aquaculture, v.200, p.1-31, 2001.

McABEE, B.J.; BROWDY, C.L.; RHODES, R.J. et al. The use of greenhouse-enclosed raceway systems for the superintensive production of pacific white shrimp Litopenaeus vannamei in the United States. Global Aquaculture Advocate, v.6, n.4, 2003.

MIAO, S.; JEN, C.C.; HUANG, C.T. et al. Ecological and economic analysis for cobia Rachycentron canadum commercial cage culture in Taiwan. Aquaculture International, v.17, p.125$141,2009$.

MOSS, S.M.; ARCE, S.M.; ARGUE, B.J. et al. Greening of the blue revolution: Efforts toward environmentally responsible shrimp culture. In: BROWDY, C.L.; JORY, D.E. (Eds.) The new wave. Baton Rouge: The World Aquaculture Society, 2001.

OTOSHI, C.A.; TANG L.R.; DAGDABAN, D. et al. Super intensive growout of the pacific white shrimp Litopenaeus vannamei: Recent advances at the oceanic institute. In: INTERNACIONAL CONFERENCE RECIRCULATING AQUACULTURE, 6., 2006, Blacksburg. Proceedings... Blacksburg: Virginia Tech University, 2006. 
OTOSHI, C.A.; NAGUWA, S.S.; FALESCH, F.C. et al. Comercialscale production of pacific white shrimp Penaeus Litopenaeus vannamei in a biosecure, super-intensive, recirculating aquaculture system. Lake Buena Vista Florida, 2008.

RESLEY, M.J.; WEBB, K.A.; HOLT, G.J. Growth and survival of juvenile cobia, Rachycentron canadum, at different salinities in a recirculating aquaculture system. Aquaculture, v.253, p.398-407, 2006.

RODRIGUES, R.V.; SCHWARZ, M.H.; DELBOS, B.C. et al. Toxicity and sublethal effects of ammonia and nitrite for juvenile cobia Rachycentron canadum. Aquaculture, v.272, p.553-557, 2007.

SAMPAIO, L.A.; SANTOS, R.; SCHWARZ, M.H. et al. Growth and survival of juvenile cobia Rachycentron canadum exposed to chronic acid stress. Proccedings... Busan: World Aquaculture 2008, 2008.

ROMBENSO, A.N.; MOREIRA, C.B.; MIRANDA-FILHO, K.C. et al. Avaliação do crescimento de bijupirá (Rachycentron canadum) alimentados com uma dieta comercial e peixe fresco. In: CONFERENCIA LATINOAMERICANA SOBRE CUltivo DE PECES NATIVOS, 3., 2009, Chascomus. Anais... Chascomus: 2009. (CD-ROM).

SAMOCHA, T.M.; LAWRENCE, A.L.; COLLINS, C.A. et al. Production of the Pacific white shrimp, Litopenaeus vannamei, in high-density greenhouse-enclosed raceways using low salinity groundwater. Journal Applied Aquaculture, v.15, n.1, p.1-19, 2004.

SAMPAIO, L.A.; ROMBENSO, A.N.; MOREIRA, C.B. et al. Crescimento de juvenis do bijupirá (Rachycentron canadum) cultivados em tanque-rede na costa do Rio De Janeiro - Brasil. In: CONFERENCIA LATINOAMERICANA SOBRE CUltivo DE PECES NATIVOS, 3., 2009, Chascomus. Anais... Chascomus: 2009. (CD-ROM).

SCHWARZ, M.H.; CRAIG, S.R.; DELBOS, B. et al. Researchindustry links assist cobia farming production. Global Aquaculture Advocate, p.56-58, 2007.

SCHWARZ, M.H.; MOWRY, D.; MCLEAN, E. et al. Performance of advanced juvenile cobia, Rachycentron canadum, reared under different thermal regimes: evidence for compensatory growth and a method for cold banking. Journal of Applied Aquaculture, v.19, p.71-84, 2007.

SCHWARZ, M.H.; CRAIG, S.R.; DELBOS, B. et al. Cobia larviculture protocols effectively replace live algae with commercially available algal paste concentrate during the greenwater phase. Journal of Applied Aquaculture, v.17, p.285-294, 2008.

SHAFFER, R.V.; NAKAMURA, E.L. Synopsis of biological data on the cobia Rachycentron canadum (Pisces: Rachycentridae). Washington, D.C.: FAO Fisheries Synopsis. 153 (National Marine Fisheries Service/S 153), U.S. Department of Commerce, NOAA Technical Report, National Marine Fisheries Service 82, 1989.

SILVA, A.F. Influência da densidade de estocagem sobre o desempenho do camarão branco Litopenaeus vannamei durante a fase final de engorda em sistema superintensivo. 2009. Dissertação (Mestrado em Aquicultura) Universidade Federal do Rio Grande, Rio Grande.

SUN, L.; CHEN, H.; HUANG, L. Effect of temperature on growth and energy budget of juvenile cobia (Rachycentron canadum). Aquaculture, v.261, p.872-878, 2006.

WASIELESKY, W.J.; ATWOOD, H.I.; STOKES, A. et al. Effect of natural production in brown water super-intensive culture system for white shrimp Litopenaeus vannamei. Aquaculture, v.258, p.396-403, 2006.

WEBB, K.A.; HITZFELDER, G.M.; FAULK, C. et al. Growth of juvenile cobia, Rachycentron canadum, at three different densities in a recirculating aquaculture system. Aquaculture, v.264, p.223-227, 2007.

WEIRICH, C.R.; STOKES, A.D.; SMITH, T.I.J. et al. Outdoor tank and pond spawning of cobia, Rachycentron canadum, in coastal South Carolina. Journal of Applied Aquaculture, v.18, p.1$16,2006$.

WEIRICH, C.R.; SMITH, T.I.J.; DENSON, M.R. et al. Pond culture of larval and juvenile cobia Rachycentron canadum in the southeastern United States: initial observations. Journal of Applied Aquaculture, v.16, p.27-44, 2004. 